\title{
Liame
}

Liame Histoire et histoire de l'art des époques moderne et contemporaine de l'Europe méditerranéenne et de ses périphéries

\section{Les États de Bourgogne à l'époque moderne : un bilan historiographique}

Jérôme Loiseau

\section{(2) OpenEdition}

Journals

Édition électronique

URL : http://journals.openedition.org/liame/71

DOI : $10.4000 /$ liame.71

ISSN : 2264-623X

Éditeur

CRISES - Centre de Recherches Interdisciplinaires en Sciences Humaines et Sociales de Montpellier

\section{Référence électronique}

Jérôme Loiseau, «Les États de Bourgogne à l'époque moderne : un bilan historiographique », Liame [En ligne], 23 | 2011, mis en ligne le 19 juillet 2011, consulté le 05 mai 2019. URL : http:// journals.openedition.org/liame/71 ; DOI : 10.4000/liame.71

Ce document a été généré automatiquement le 5 mai 2019.

Les contenus de Liame sont mis à disposition selon les termes de la Licence Creative Commons Attribution - Pas d'Utilisation Commerciale - Pas de Modification 4.0 International. 


\title{
Les États de Bourgogne à l'époque moderne : un bilan historiographique
}

\author{
Jérôme Loiseau
}

1 «Avant 1789, la Bourgogne était le seul pays du royaume qui, avec l'Artois, la Bretagne, le Béarn, le Cambrésis, le comté de Foix, le Languedoc et la Provence, fût resté en possession du privilège, envié partout ailleurs, de consentir l'impôt et de s'administrer lui-même ». Voilà comment l'archiviste du département de la Côte-d'Or, Joseph Garnier, invitait le curieux, au début de la Troisième République, à découvrir les archives de cette institution abolie lors la Révolution française (1790) et qui demeure comme le seul antécédent à nos conseils régionaux d'aujourd'hui. Le mérite principal de Garnier est de définir brièvement le cœur de la fonction des États : l'impôt.

2 Plus précisément, ils avaient pour tâche de le concéder à la monarchie, de le répartir entre les Bourguignons et de le lever. Autrement dit, le rôle de l'assemblée était nodal et l'autonomie de la province de Bourgogne notable. Le mécanisme fiscal s'enclenchait par une convocation royale qui prenait la forme de lettres particulières que le greffier, un officier des États expédiait à ses membres une fois que le gouverneur lui en avait donné l'ordre. La réunion, triennale, se tenait à Dijon depuis le XVI ${ }^{\mathrm{e}}$ siècle, sauf empêchement majeur. Une séance d'ouverture rassemblait les députés des ordres pour entendre les demandes royales pour les trois années à venir. Le gouverneur puis le premier président du parlement s'exprimaient pour les présenter et les justifier. À l'issue de ces harangues, les députés se réunissaient par ordre. La première séance était consacrée au choix de leur élu respectif qui représenterait l'ordre pendant la durée des États et jusqu'à la tenue suivante. Les séances ultérieures étaient consacrées à débattre des demandes royales et à évoquer les problèmes financiers et administratifs. Après une quinzaine de jours, une fois que les avis avaient été pris, les trois ordres étaient de nouveau rassemblés, en conférence. Les décisions de chaque ordre étaient alors transformées en décret des États. Un cahier de doléances était également rédigé. Il devait être porté par les élus des trois 
ordres en voyage d'honneur, à Versailles où le roi en prenait connaissance et y répondait. Les États avaient donc deux fonctions : l'impôt et la représentation de la province.

Une fois revenus de Versailles, les élus s'appliquaient à organiser les opérations fiscales. La base en était le feu auquel était attachée une cote. Chaque communauté était imposée pour un nombre précis de feux. Il revenait ensuite aux collecteurs de répartir entre les habitants, en fonction de leur richesse supposée, la charge fiscale. Cette dernière était collectée à l'échelle des cinq bailliages principaux par les trésoriers, qui remettaient ensuite les sommes entre les mains du trésorier général des États. Cet officier des États tenait également les comptes des revenus indirects de la province, notamment les crues sur le sel et les octrois de la Saône et des emprunts. Il était responsable de l'effectuation des paiements au Trésor royal ainsi que de ceux qui étaient réalisés à l'intérieur de la province : remboursement des étapes, marchés publics, arrérages et principaux de rentes. Ces paiements étaient ordonnés par les États, en vertu de leurs décrets, mais aussi par le bureau des élus. Cette commission permanente des États était composée de l'élu du clergé, de celui de la noblesse et de deux élus du tiers état, le vicomte-mayeur de Dijon et le maire d'une autre ville de Bourgogne selon un tour de rôle bien établi. À ces quatre membres s'ajoutaient trois officiers : l'élu du roi et deux autres émanant de la Chambre des comptes. Leur administration, c'est-à-dire l'emploi des deniers provinciaux, faisait l'objet d'un examen critique par la commission des alcades, députés des États nommés par leur ordre lors de la tenue précédente: deux clercs, deux nobles et trois membres du tiers. Un cahier de remarques était alors rédigé; il en était fait lecture dans chaque chambre. Ainsi existait-il un contrôle des comptes opéré à la fois par les officiers du roi au sein du bureau des élus et par les représentants des États.

Voilà donc brossée à grands traits la trame institutionnelle de l'assemblée des trois ordres du duché de Bourgogne. Elle tient davantage de l'assemblée bretonne que de l'assemblée languedocienne ou de celle du Comminges qui se réunissaient chaque année. Quelle en fut donc la fortune historiographique?

\section{Légende dorée contre légende noire (1775-1890)}

5 Le premier historien à livrer une analyse du rôle des États dans l'administration provinciale fut l'abbé Courtépée; son histoire «totale» de la Bourgogne, dont la publication s'échelonna de 1775 à 1788, ne comporte pourtant qu'une dizaine de pages traitant de l'institution' ${ }^{1}$. Seul un court paragraphe revêt les qualités d'une analyse historique. À dire vrai, il s'agit pour l'essentiel d'un panégyrique de l'action de l'assemblée, résumée par deux puissantes interrogations : «quels services n'ont pas rendu les États à la province par leur union, leur crédit et leur zèle? » et « quels établissements utiles n'ontils pas favorisés?" Animés par un zèle qualifié de "patriotique», les États ont successivement protégé la province d'un excès d'impôt au temps de Mazarin, encouragé l'établissement des manufactures, fondé l'école de dessin sans oublier qu'ils établirent de véritables chemins dans la province. Courtépée construisait par ces quelques remarques une véritable légende dorée de l'assemblée, prenant sa source davantage dans des arguments d'autorité que dans une présentation raisonnée de leur œuvre administrative.

6 La réplique à cette vision idéale et très succincte fut amenée, longtemps après la Révolution, par Alexandre Thomas. En 1844 paraissait en effet sa thèse d'histoire du droit intitulée : Une province sous Louis XIV. Situation politique et administrative de la Bourgogne de 1661 à 1715². L'année suivante Amédée Tardieu en livrait une recension éclairante: «Ce 
livre, je le dis sans la moindre pensée de blâme, ne présente aucun des caractères d'une thèse universitaire: l'auteur s'y est proposé pour objet la politique plutôt que l'histoire, et s'y est révélé par des qualités d'esprit plutôt pratiques que spéculatives ${ }^{3}$ ». À la légende dorée succédait alors la légende noire des États. Thomas est un patriote et un républicain convaincu que la grandeur de son pays tient à l'unité de son peuple et de son État. Cette unité est analysée comme le résultat d'un processus historique au cours duquel la monarchie joua un rôle indéniable mais mineur en comparaison de la dynamique révolutionnaire qui se leva en 1789. "Huit cents ans de labeurs n'ont peut-être pas produit de plus grandes choses que nos cinquante années de merveilles $»^{4}$ écrit-il dans son introduction; ce à quoi, un lecteur répliqua, dans le volume que nous avons consulté, par un «tu parles!» admirablement calligraphié au crayon de papier dans la marge. L'ouvrage de Thomas s'inscrit donc dans le débat politique du premier XIX ${ }^{e}$ siècle qui voit s'affronter partisans et opposants de la Révolution française. Les États de Bourgogne deviennent alors un élément de l'argumentation en faveur de la disparition de l'Ancien Régime. Sous la plume de Thomas, ils sont décrits comme une force "de résistance et d'isolement " réclamant contre l'activité de la «centralisation, sous prétexte qu'elle était le despotisme et qu'ils étaient la liberté. Liberté menteuse ! ${ }^{5}$ ». Ils deviennent alors l'obstacle le plus évident à l'unité nationale, " honneur et force de la France». Ils incarnent le parti de la «divergence des privilèges», causes de la " ruine ${ }^{6} \gg$ du pays. Pour l'auteur, les institutions provinciales participaient pleinement de la fragmentation politique de la France. Elles nourrissaient donc l'absolutisme royal qui lui seul disposait de suffisamment de force - d'un privilège plus élevé - pour faire taire l'égoïsme des libertés. Réhabiliter les États provinciaux revenait donc à faire renaître la société du passé ; inversement, les abaisser permettait de conforter la société nouvelle. Alexandre Thomas s'est donc employé à montrer combien l'assemblée de Bourgogne était un aréopage inégalitaire de privilégiés, dont la seule ambition était de résister aux progrès de l'autorité royale. Élus et alcades de la province connurent un sort particulier sous la plume de Thomas, qui en fit des hommes peu vertueux, négligents, impuissants et parfois incompétents.

7 Une génération plus tard, Joseph Garnier reprit à son tour cette question dans son introduction à l'inventaire de la Série $\mathrm{C}$ où sont archivés les papiers des États ${ }^{7}$. Si l'homme d'archives semble rendre grâce à l'activité des États, retrouvant des formules à la Courtépée, le fonctionnaire de la République naissante l'emporte. Tournant le dos à l'incurie des États, il incrimine au contraire l'absolutisme destructeur des libertés provinciales. Louis XIV, comme chez Tocqueville ou Lavisse, fit régner le silence dans l'assemblée. Destitués de leurs responsabilités, les députés des trois ordres ne pouvaient que se chamailler et déchoir en enfance : "l'activité de nos députés n'a plus pour s'exercer que des questions de préséance ou d'étiquette; aussi dans chaque ordre, quel assaut d'envie, de jalousie d'exclusivisme $e^{8}$ ». La figure tutélaire de l'intendant point alors dans ce discours historique. La centralisation monarchique a brisé l'indépendance provinciale, voilà ce que Garnier nomme « loi fatale ", concluant son propos d'une touche romantique : «c'est à peine si l'on ose murmurer ${ }^{9} \%$. Les États étaient devenus une coquille vide, après avoir été successivement la quintessence d'une administration provinciale puis un repaire de notables corporatistes. 


\section{Les États, un objet d'histoire froid (1932-1988)}

8 Cette vision morale des États, qui se comprend dans le contexte de l'affirmation et de l'enracinement de l'idée républicaine, ne fut dépassée que tardivement, en 1932, par la parution de l'ouvrage que Joseph Billioux consacra aux origines médiévales de l'institution ${ }^{10}$. Jusqu'alors, les historiens des États s'étaient essentiellement intéressés à la période moderne, et plus précisément au Grand Siècle, se contentant de jeter un voile d'ancienneté sur leurs origines. Avec Billioux, le vent méthodique se lève et la critique historique construit un nouveau discours qui fait une place au doute, comme l'indique l'introduction générale. "Malheureusement, l'histoire des États de Bourgogne est celle d'une institution qui a laissé des témoignages si rares et si dispersés de son activité parlementaire qu'elle ne saurait être abordée sans un sentiment de profonde modestie ${ }^{11} »$. On doit à cette modestie une démarche scientifique imparable qui, en postulant diverses hypothèses scrupuleusement vérifiées en regard de la documentation disponible, débouche sur un véritable discours des origines. Ainsi apprend-on que les Etats sont sortis de la curia ducis et que leur première assemblée date de 1352. Elle avait pour objet d'agréer à l'impôt royal de six deniers par livre. L'origine des États se trouve donc dans l'insuffisance des ressources domaniales ordinaires de la royauté, qui l'oblige à requérir de ses vassaux et d'une province provisoirement entre ses mains un secours extraordinaire. Création royale passée dans les mains ducales, les États ne peuvent être convoqués que par le souverain. Une telle affirmation contredisait toutes les analyses précédentes en prouvant que la monarchie et les États étaient finalement les deux faces d'un même pouvoir, liées par un contrat politique : "les États comprirent dès 1371 que la réforme des exactions fiscales était plus utile au pays qu'une vaine résistance aux requêtes des Ducs ${ }^{12}$ ». Sans la formuler expressément, la nature transactionnelle des États était esquissée : l'impôt contre la tranquillité publique. Le reste du travail est une enquête minutieuse sur les soubassements institutionnels. Résumons-les. Dijon ne l'emporta comme capitale de l'assemblée qu'à partir de 1386. Il n'y avait pas d'intervalle régulier entre les sessions avant 1500. Les élus n'apparurent qu'en 1384 pour lutter contre les exactions des baillis et des commissaires ducaux lors de la perception de l'impôt. Ils étaient jusqu'en 1438 choisis alternativement par le duc et les États, mais, depuis 1410, le duc adjoignait à la commission des élus un maitre des comptes. En 1455, la Chambre des comptes députait un de ses membres comme adjoint de l'élu ducal. Très finement, Billioux comprit aussi que les élus cessèrent - à mesure que l'institution se développait - d'être des délégués de fortune pour devenir «de vrais administrateurs du pays spécialisés et permanents ${ }^{13}$ ». Ainsi ouvrait-il la voie à une histoire plus rationnelle des États de Bourgogne. Sa démarche trouva un large écho au sein de la revue des Annales de Bourgogne, dont la fondation en 1929 avait été presque concomitante de la parution de son livre, ainsi que dans les Mémoires pour l'histoire du droit et des institutions des anciens pays bourguignons, comtois et romands et plus encore.

9 L'histoire des États allait devenir une histoire véritablement érudite délaissant toute considération politique afin d'être, selon le mot de Thucydide, une acquisition pour toujours. Sans prétendre à l'exhaustivité - il faudrait pour cela au moins inventorier les tables analytiques des deux revues citées plus haut -, il est possible de dégager les grandes orientations de la recherche, le plus souvent menée par des acteurs locaux. Un premier centre d'intérêt regroupe des communications portant sur la géopolitique des 
États, d'où il ressort que l'institution provinciale absorba régulièrement les États particuliers d'Auxonne $(1638)^{14}$, d'Auxerre $(1668)^{15}$, de Bar-sur-Seine (1721) et du Charolais $(1751)^{16}$. Ne restaient comme États particuliers, à la veille de la Révolution, que ceux du Mâconnais. L'extension géographique s'accompagna aussi de transferts de compétences juridiques de l'État vers les États ${ }^{17}$. D'une façon plus générale, la question des rapports avec l'autorité royale fut posée ${ }^{18}$. L'accent fut également mis sur les élus et le personnel des États. Soit que les attaques d'Alexandre Thomas sur la probité et les compétences de ces administrateurs aient incité les historiens à les étudier, soit que la documentation les concernant fut abondante, cette entrée dans l'histoire des États est indéniablement l'une des plus importantes, qu'ils fussent considérés pour eux-mêmes ${ }^{19}$ ou dans leurs rapports avec les autres autorités provinciales ${ }^{20}$. Un autre centre d'intérêt tout aussi important fut la question fiscale et financière, notamment pour le siècle des Lumières $^{21}$. C'est d'ailleurs sur ce sujet que se retrouve l'esprit incisif à l'égard de l'institution, si courant dans l'historiographie du siècle précédent. On le doit à Georges Bouchard qui, en étudiant l'administration financière, pointa quelques difficultés en regard des normes comptables actuelles : «Les comptes de la Bourgogne se présentent dans un état parfait de tenue et de conservation. Seulement on y relève tout de suite des hérésies comptables qui suffiraient aujourd'hui à faire ajourner un candidat aspirant à remplir à tire provisoire les fonctions de sur-numéraire adjoint dans quelque bureau de finance. L'erreur de principe qui tombe tout de suite sous les yeux est d'inscrire en recette les emprunts au même titre que les impôts $»^{22}$. Il faut comprendre que les emprunts étaient inscrits non comme une dette mais comme une ressource dans le cadre d'une comptabilité, à double partie recettes et dépenses, qui ignorait, par conséquent le chapitre de l'endettement public. Cette étrangeté comptable avait le mérite de taire la dette et donc de conforter le crédit des États ${ }^{23}$. Bouchard exagéra toutefois le trait en évoquant une sorte de "putréfaction administrative $»^{24}$ pour qualifier la gestion financière du bureau des élus, qui était loin de mériter une telle sentence, comme le prouve l'existence d'un document remarquable, établi à la demande même des Élus en 1782. Ce document reprend l'ensemble des impositions et des dettes de la province sous la forme d'un tableau synoptique ${ }^{25}$ afin, justement, de réordonner la gestion des comptes publics.

10 Outre ces questions techniques, la recherche considéra aussi le cérémonial des États, tentant de faire revivre une institution perdue de vue. Cette approche permettait d'appréhender ce qu'était la puissance publique provinciale, c'est-à-dire l'appareil par lequel elle se donnait à voir et représentait la province à Dijon, dans le cadre des tenues des États ${ }^{26}$, ou à Versailles lors du voyage d'honneur triennal ${ }^{27}$. À ce titre, il convient de mentionner le travail de Christine Lamarre ${ }^{28}$ concernant les dons en nature que les États faisaient, sur le vote des députés, à l'ensemble de l'appareil monarchique. Ces véritables " pots-de-vin » constituaient l'un des langages de la politique à l'âge moderne et de façon donc très officielle. Néanmoins, au temps des Annales E.S.C., le parent pauvre de l'historiographie des États est bien l'histoire économique en dépit d'une contribution de l'historien Daniel Ligou sur l'amélioration du réseau viaire au XVIII ${ }^{e}$ siècle $^{29}$.

11 Finalement, les États sont devenus un objet historique dépassionné en général mais éclaté en différentes contributions relevant de divers champs historiques : l'histoire du droit, l'histoire administrative - notamment financière - et l'histoire politique et sociale. La synthèse, sous la direction de Jean Richard, vint en 1978 mais il s'agissait d'une histoire générale de la province au sein de laquelle la question des États, traitée par Daniel Ligou, était minime ${ }^{30}$ : description de l'assemblée, de ses fonctions et des grands traits de son 
histoire. Le seul apport vraiment novateur en regard des autres histoires de la Bourgogne ${ }^{31}$ était la mention que la tenue des États était un moment brillant de la vie sociale dijonnaise. L'interrogation majeure de l'auteur portait sur l'indépendance de l'institution en mettant en cause son « recrutement caricatural » et des « délibérations sous le contrôle du roi et du Parlement ». Il notait également la montée en puissance des États après 1740 et leur reconnaissait un rôle d'interlocuteur de la monarchie en Bourgogne, notamment à travers les élus, dont les responsabilités dans le domaine économique et dans celui des travaux publics étaient soulignées. Ainsi étaient esquissées des pistes de recherche qui furent suivies la décennie suivante par des historiens de langue anglaise.

\section{« La manière anglo-américaine » (1980-2006)}

Le socle de l'enquête anglaise est constitué de l'extraordinaire synthèse de James Russell Major sur les États Provinciaux en France de la Renaissance à Louis XIV ${ }^{32}$. À la différence des travaux précédents, le propos de l'auteur est de situer les États dans leurs rapports avec la monarchie sans rien ignorer des caractères particuliers de chacune des assemblées provinciales. Ce faisant, le critère de la fiscalité est introduit comme facteur dynamisant l'histoire des assemblées. Dans l'esprit de J. R. Major, les impôts deviennent le révélateur du processus de construction de l'État moderne. Les États provinciaux sont alors un poste d'observation des résistances à l'État, fondées principalement sur le respect des privilèges provinciaux, et des stratégies royales pour parvenir à soutirer davantage de ressources pour financer les guerres. On doit alors à cette approche d'avoir isolé un mode particulier de relations politiques entre le centre royal et les périphéries provinciales que J. R. Major nomme «marchandage général ». Une fois la Fronde passée, il sera perçu comme dégradant la majesté du jeune Louis XIV, animé d'une prétention absolutiste à nulle autre pareille. Cette perspective sera revisitée dans un second ouvrage ${ }^{33}$, d'où il ressort que l'œuvre de Louis XIV doit beaucoup aux entreprises de Louis XIII et de Richelieu, ce qui atténue grandement l'idée d'une rupture historique entre les deux règnes. Il reste que l'œuvre de J. R. Major ouvrit des perspectives nouvelles, notamment sur la manière dont les États s'acquittèrent des sommes consenties à la monarchie. Trois possibilités existaient: une augmentation des impôts directs, un relèvement des taxes indirectes ou l'emprunt.

Éclaircir cette question fut le travail de deux chercheurs : Mark Potter et Jean-Laurent Rosenthal ${ }^{34}$. Dans une série d'articles, ils montraient que les États avaient choisi les taxes indirectes et l'emprunt pour répondre aux exigences fiscales de la monarchie. Les revenus du sel et les octrois acquittés pour naviguer sur la Saône constituaient les fonds garantissant les émissions de rentes provinciales. Selon eux, les emprunts passèrent de trois millions et six cent mille livres pendant la décennie 1680-1689 à plus de six millions durant la guerre de Neuf ans, puis de douze à quinze millions de livres pendant les deux premières décennies du XVIII siècle $^{35}$. Durant la même période, la taille royale, financée par les levées sur les produits de la terre, augmenta moins, restant en moyenne inférieure à un million de livres par an. Les emprunts augmentèrent donc deux fois plus vite que l'imposition directe. Les détenteurs de la dette publique furent d'abord les élites locales. Entre 1660 et 1709, les trois quarts des prêts étaient conclus par des Bourguignons, mais après 1740 , plus de la moitié (54 \%) se faisait à Paris. Cette première découverte de nature économétrique fut prolongée par une analyse politique menée par M. Potter. Considérant que le marché du crédit est l'image financière des relations de clientèle, cet historien 
perçoit les États comme une arène idéale pour construire des coalitions parmi les élites. Ainsi, les États jouent un rôle crucial en organisant les relations entre la cour et la province ${ }^{36}$. L'essentiel du propos consiste à détailler le mécanisme financier par lequel les États surent s'imposer et faire face à la monarchie et à la clientèle des Condé dans la province. Ils agirent pour maintenir les privilèges bourguignons et protéger l'élite locale de la politique royale, notamment par le rachat des édits créant de nouveaux offices ${ }^{37}$. L'expression clé pour comprendre les relations entre la monarchie et les États provinciaux est celle de "coopération contrainte ${ }^{38}$. Elle est la synthèse de la complexité des liens qui se tissèrent à partir du règne personnel et qui dépassèrent la simple intermédiation des Princes de Condé. L'institution fut en capacité de s'intégrer pleinement dans le jeu monarchique. Les deux pôles produisirent alors une politique d'accommodement réciproque qui permit de maintenir les élites locales loyales à la monarchie et à la monarchie de financer ses guerres. La conclusion est nette : « toutes les indications de la seconde moitié du règne de Louis XIV prouvent que le pouvoir royal et le pouvoir des élites sont interdépendants et le pouvoir de l'État était fondé sur cette interdépendance ${ }^{39}$. Les anciennes conceptions d'Alexis de Tocqueville ${ }^{40}$ dans L'Ancien Régime et la Révolution ou d'Ernest Lavisse dans son Louis XIV ${ }^{41}$ sont non pas seulement nuancées mais bien contredites. Les États provinciaux, singulièrement ceux de Bourgogne, ne furent pas des coquilles vides. Bien au contraire, ils furent, en généralisant le cas bourguignon, des assemblées politiques par excellence. Ce point fut aussi démontré par l'historienne américaine Beth Nachison qui consacra sa thèse au gouvernement des Princes de Condéet ${ }^{42}$ Principalement consacré à la question du patronage, le travail aborde les États provinciaux à travers le contrôle que les Princes de Condé, gouverneurs depuis 1632, exerçaient sur la nomination des élus des trois ordres ainsi que sur les officiers des États : secrétaire, trésoriers, greffiers, et procureurs syndics. Or, après le traité des Pyrénées (1659), si le gouvernement Bourgogne fût rendu à Louis II de Condé, force est de constater que le Prince n'y séjournait que pour la durée des États. Ce handicap, en apparence, fut compensé par la mise en place d'un vaste réseau de clients, administré directement en Bourgogne par l'intendant du prince, Thésut de Ragy ${ }^{43}$.

14 Autrement dit en faisant porter l'analyse d'une part sur le système fiscal et la dette publique et d'autre part sur les liens de clientèles, la "manière anglo-américaine » d'écrire l'histoire des États de Bourgogne mit en exergue les capacités politiques propres de l'institution quand ces dernières avaient été étouffées, méjugées ou simplement esquissées.

15 Mais le travail le plus abouti est incontestablement celui de Julian Swann ${ }^{44}$ qui s'inscrit dans ce courant "fisco-financier» appliqué naguère par William Beik ${ }^{45}$ au cas languedocien tout en restituant l'œuvre de l'institution au siècle des Lumières. Il offre la première synthèse de la vie administrative en reprenant le dossier du personnel et du fonctionnement des États. En outre, il réhabilite largement le travail des élus en soulignant l'amélioration de leur administration, notant que « les péchés des élus » avaient été exagérés, soulignant, à l'appui de sa démonstration, "un enthousiasme réformateur ${ }^{46}$ " dans les dernières années du siècle. Il pointe alors la réorganisation fiscale afin d'améliorer la perception de la taille et accroître la participation des privilégiés à la capitation et au vingtième. Il ne sombre pas dans l'angélisme et indique clairement les limites de cette réforme comme le refus d'établir un cadastre, synonyme d'une meilleure connaissance des propriétés et d'un éventuel passage à la taille réelle. Cette administration n'était donc pas aussi "archaïque qu'on l'a écrit ${ }^{47}$ ». Bien au contraire 
poursuit Swann, elle témoigne d'un processus d'« enlightenment " ${ }^{48}$ à l'œuvre dans la politique de désenclavement de l'espace bourguignon par la route et les canaux du centre, de Bourgogne et de Franche-Comté dont le percement débuta à partir de 1778. Les élus se saisirent également, après la faillite de l'étapier général en 1749 , des étapes de la province. Les questions agricoles, notamment celle des enclosures sont débattues. Les États encouragent le développement de nouvelles cultures comme les fruitiers (1724), en particulier le mûrier. L'auteur dresse un bilan tempéré de l'action des élus et des États montrant notamment que pour ce qui concerne le commerce, l'éducation, la lutte contre la pauvreté et la mendicité, l'œuvre était plus timide. Au final, l'idée d'un bilan globalement négatif ne se soutient plus. Les États furent des auxiliaires fidèles de l'administration royale et virent leur pouvoir croître particulièrement après 1750 . Cette densification du rôle des États introduit au problème de leur niveau d'autonomie. L'auteur la voit par excellence dans le rachat régulier des édits bursaux et dans la capacité à conclure des abonnements fiscaux. Cette politique permettait aux députés des États de maintenir le compromis «fisco-financier» qui s'est dégagé sous Louis XIV et ainsi protéger "l'existence de rentiers des élites provinciales ${ }^{49}$ ». C'est bien en ce point que la fragilité des États était manifeste: en renforçant les privilégiés, ils s'affaiblissaient, méritant alors l'appellation de « géant aux pieds d'argile ${ }^{50}$ ».

L'historiographie anglo-américaine a donc considérablement fait avancer la connaissance sur les États de Bourgogne. Au cœur de l'analyse se trouvent les notions de finance et de patronage ; elles débouchent sur la mise en évidence d'un cercle vertueux mis en branle à partir de 1658 emportant la province dans un tourbillon d'obéissance. Mais, moins qu'une réduction passive à l'obéissance, il s'est agi davantage d'une servitude volontaire motivée par l'intérêt collectif et personnel. Louis XIV proposa in fine un destin politique et des récompenses. Loin d'être annihilés par l'absolutisme triomphant, les États furent au contraire réinventés.

\section{Les perspectives récentes (2006-2010)}

17 Dans la trame de ces acquis historiographiques, la recherche récente s'oriente vers un approfondissement de la connaissance sur les États provinciaux. Le premier exemple est fourni par la thèse de Charles Papon ${ }^{51}$. L'ouvrage, très savant, propose à la fois une histoire de l'institution des origines modernes jusqu'au règne de Louis XIV et une analyse du système fiscal et financier mis en place après 1658. D'une grande érudition, le travail permet de situer chacun des acteurs dans un contexte historique qui lui donne sens. Tout comme Swann, Papon décrit une assemblée qui est aux antipodes d'un corps politique dévitalisé : "se dessine ainsi une politique de la monarchie vers le pouvoir local qui déborde largement la simple demande de consentement aux impôts royaux et qui est loin d'être formelle. Dans certains domaines, l'assemblée reste un interlocuteur à part entière du gouvernement avec des contraintes propres au fait financier dans la France moderne ${ }^{52}$ ». Sur ce dernier point, Papon souligne avec force que les élus devinrent les acteurs essentiels du gouvernement financier de la province, forgeant l'expression pour les désigner de « commission exécutive triennale $^{53}$ » étroitement liée au contrôle général des finances. La montée en puissance des élus a pour corollaire un affaiblissement de la représentation provinciale gênée par le rythme triennal qui aurait empêché ses membres de s'intéresser véritablement aux affaires provinciales et d'y prendre une part active. Le voyage d'honneur est alors compris comme une manière de neutraliser les doléances provinciales ${ }^{54}$ puisqu'il fallait à 
l'assemblée attendre près de trois ans la réponse à ses remarques. Cette division de l'espace public entre fonctions exécutives et fonctions représentatives découle du coup de force que Louis XIV opéra en 1659. Il eut pour conséquence le passage de l'impôt octroyé par manière de don, selon les privilèges bourguignons, à l'impôt permanent et levé d'autorité. Ce passage s'est effectué dans l'extraordinaire ${ }^{55}$, c'est-à-dire pour la partie des impôts qui ne connaissait pas d'imputation. La complexification des affaires financières en fut la conséquence. Elle requérait de la part des élus une compétence financière notable qui ne pouvait être portée par l'assemblée tout entière. C'est ainsi qu'il faut comprendre que le vote de l'impôt devint "une convention politique (...) au service d'une élite ${ }^{56}$ ». L'auteur affine alors la compréhension du compromis politique mis à jour par Mark Potter en clarifiant la question de l'assiette fiscale. En montrant que le financement des rentes provinciales reposait sur les revenus tirés des octrois de la Saône - les trois quarts en étaient reversés à la province durant la guerre de Neuf ans - et des crues sur le sel dont seuls les officiers du roi et le personnel des États étaient exemptés, il prouve que le tiers état gagnait aussi à cet arrangement en ce qu'il n'était pas seul à supporter le fardeau fiscal ${ }^{57}$.

Percer la mécanique du pouvoir semble être aussi le but de l'ouvrage que Stéphane Pannekoucke consacre aux Condé comme gouverneurs de Bourgogne ${ }^{58}$. L'intention de la recherche est de saisir le gouverneur en actes et dans ses rapports avec les autres formes de pouvoir dans la France d'Ancien Régime. Ce faisant, l'auteur consacre un chapitre aux États de Bourgogne ${ }^{59}$ dans lequel il brosse une description mise à jour de l'institution. Son apport est de détailler la manière très administrative dont use le prince pour faire l'élu du clergé et celui de la noblesse ${ }^{60}$ bien avant la tenue des États. Cette dernière voyait aussi l'influence du prince s'exercer pleinement dans le cadre de la conférence, «organe officieux » qui réunissait deux fois par jour autour du prince «quelques-uns des personnages les plus influents aux États ", médiateurs naturels entre le gouverneur et les députés de la province. Les officiers des États étaient aussi associés à ces réunions. Un tel dispositif était censé encadrer le travail de l'assemblée et éviter le retour des frondes fréquentes avant 1674. Les princes témoignaient là d'un grand savoir-faire puisque hormis la dernière assemblée, du 12 novembre au $1^{\text {er }}$ décembre 1787, elles furent toutes marquées par une réelle quiétude.

Les deux travaux confirment largement les orientations historiographiques précédentes. Ils en constituent un approfondissement notable.

\section{Conclusion}

Les États de Bourgogne, loués puis décriés, sont au tournant du XXe siècle devenus un objet historique dépassionné. Leur défense et illustration ne conditionnent plus la pertinence de la Révolution et de la République en France. Pendant longtemps, ils restèrent un objet d'histoire locale grâce notamment à l'activité du professeur Ligou, dont nombre de remarques se vérifièrent lorsque les historiens anglo-américains s'en saisirent. Porteurs de nouvelles questions - les finances et le clientélisme principalement -, ils firent davantage l'histoire de l'État en France en se servant des États comme d'un révélateur. Cette manière de faire réinventa l'objet historique mettant en scène un pouvoir supposé absolu mais dont la force résidait dans la capacité à faire advenir des compromis acceptables par toutes les parties. Contrôlés par la monarchie, mus par la 
volonté royale et l'intérêt de ses députés, les États ne furent-ils finalement que cette créature collective disposée au consentement?

Répondre à cette question supposerait d'approfondir encore l'histoire des États mais davantage pour eux-mêmes qu'en tant que simples reflets de l'État moderne de telle sorte que puissent être repérées des pratiques politiques nouvelles qui n'ont pas, à coup sûr, manqué d'émerger une fois l'opposition à une monarchie briseuse des privilèges devenue archaïque. Les États sont encore un champ historique fécond pour l'histoire de la décentralisation en France en tant que première et seule expérience de pouvoir décentralisé de l'Ancien Régime. Enfin, la connaissance des États gagnerait à une mise en comparaison avec les autres assemblées représentatives de France. Gageons qu'un autre visage de l'histoire de France en serait dessiné.

\section{NOTES}

1. Claude COURTÉPÉE et Edme BÉGUILLET, Description générale et particulière du duché de Bourgogne, Dijon, chez V. Lagier, 1847-1848, 4 vol. ; 3e éd., Lyon, éd. Horvath, 1976, vol.1, p. 324-334.

2. Alexandre-Gérard тномAS, Une province sous Louis XIV. Situation politique et administrative de la Bourgogne de 1661 à 1715, d'après les manuscrits et les documents inédits du temps, Paris, Joubert, 1844, 458 p.

3. Bibliothèque de l'École des Chartes, 1845, t.6, p. 76-79.

4. Alexandre-Gérard THомAs, Une province... op. cit., p. 6.

5. Ibid., p. 6 et 7.

6. Ibid., p. 19.

7. Joseph GARNIER, Inventaire sommaire des archives départementales antérieures à 1790, Côte-d'Or. Série

C, États du duché de Bourgogne, comtés et pays adjacents, Dijon, Darantière, 1886-1890, t. III.

8. Ibid., p. 11.

9. Ibid., p. 14 .

10. Joseph BILlioud, Les États de Bourgogne aux XIV et XV siècles, Dijon, Académie des Sciences, Arts et Belles lettres, 1932, 498 p. La même année paraissait une étude similaire sur les États de Bretagne : Armand REBILLON, Les États de Bretagne de 1661 à 1789. Leur organisation, l'évolution de leurs pouvoirs, leur administration financière, Paris, Auguste Picard et Rennes, Plihon, 1932, 825 p.

11. Joseph BILLIOUD, Les États...op. cit., p. 8.

12. Ibid., p. 200.

13. Ibid., p. 253.

14. François MOREAU, «La suppression des États du comté d'Auxonne et leur réunion aux États du duché de Bourgogne", Mémoires pour l'histoire du droit et des institutions des anciens pays bourguignons, comtois et romands, t. 2, 1935, p. 189-194.

15. André Guillors, «La fin d'un pays adjacent. L'union du comté d'Auxerre aux États de Bourgogne. $1668 »$, Annales de Bourgogne, t. 33, 1961, p. 5-26.

16. Jean RICHARD, «Le Charolais et la réunion de 1684 », Annales de Bourgogne, t. 56, 1984, p. 5-18; Louis LAROCHE, «Les États particuliers du Charolais", Mémoires pour l'histoire du droit et des institutions des anciens pays bourguignons, comtois et romands, 1939, t. 6, p. 145-194. 
17. Léon BLIN, «La juridiction des eaux et forêts sur la Saône transférée aux élus de Bourgogne, 1781 ", Mémoires pour l'histoire du droit et des institutions des anciens pays bourguignons, comtois et romands, 1972, t. 31, p. 157-165 ; «L'accession des États de Bourgogne à la juridiction de la voierie, 1703-1708 », Congrès de l'association Bourgogne des Sociétés Savantes, Beaune, 1974.

18. L. MADON, " Les relations entre le gouvernement royal et la Bourgogne d'après la correspondance du comte de Saint-Florentin, secrétaire d'État chargé des affaires de la province de 1740 à 1750 ", Mémoires pour l'histoire du droit et des institutions des anciens pays bourguignons, comtois et romands, 1939 , t. 6, p. 247-250.

19. Pierre BODINEAU, "Aux origines des fonctionnaires régionaux: la politique du personnel administratif des États de Bourgogne ", Mémoires pour l'histoire du droit et des institutions des anciens pays bourguignons, comtois et romands, 1990, p. 147-161 ; François DUMONT, « L'élu du roi aux États de Bourgogne ", in Droit privé et institutions régionales. Études offertes à Jean Yver, Paris, P.U.F., 1976, p. 197-207 ; Daniel LIGOU, "Élus et alcades des États de Bourgogne au XVIII ${ }^{e}$ siècle ", Actes du $92^{e}$ Congrès des Sociétés Savantes, Strasbourg et Colmar, Paris, Bibliothèque nationale, 1967, p. 19-40.

20. Daniel LIGou, «Les élus généraux de Bourgogne et les charges municipales de 1692 à 1789 ", Actes du $90^{e}$ Congrès des Sociétés Savantes, Nice, Paris, Bibliothèque nationale, 1966, p. 95-119; « Le personnel de l'intendance et des États de Bourgogne : aux origines du fonctionnaire moderne ", Actes du $111^{e}$ Congrès National des Sociétés Savantes, Poitiers, Paris, éd. du CTHS, 1987, p. 67-77.

21. Daniel LIGou, "Les États de Bourgogne et les problèmes fiscaux à la fin du XVIII siècle ", Mélanges Antonio Marongiu, Bruxelles, Éd. de la librairie encyclopédique, 1968, p. 97-128 ; «Un impôt en Bourgogne sous l'Ancien Régime: les vingtièmes ", Actes du $91^{e}$ Congrès des Sociétés Savantes, Rennes, Paris, Imprimerie nationale, 1969, p. 183-215.

22. Georges BOUCHARD, «Comptes borgnes et fiscalité aveugle », Annales de Bourgogne, t. 26, 1954, p. 13.

23. Voir sur ce sujet notre article, «Les comptes des États de Bourgogne, entre prescriptions monarchiques et règlements provinciaux (fin $\mathrm{XVI}^{\mathrm{e}}$ - fin XVIII ${ }^{\mathrm{e}}$ siècles) », in Les comptes publics : enjeux, pratiques, modèles de 1500 à 1850, actes du colloque de Nanterre, 10-12 juin 2010, Rennes, P.U.R., 2011 (à paraître).

24. «Plusieurs penseront sans doute que nos attaques contre l'administration de l'Ancien Régime ne sont qu'une apologie implicite pour une autre forme de gouvernement et que nous devons réciter chaque soir, en forme de prière quelques versets de l'évangile républicain selon saint Mathiez. Il n'en est rien. [...] Pour qui ne veut rien démontrer l'étude de l'histoire conduit à un scepticisme souriant qui se transforme par degrés en athéisme politique. Cette dernière forme de la pensée permet à la fois la douceur de louer et le recours aux pires des finances, annonçait le déplacement prochain des suzerainetés sociales. Parmi les grands de l'heure, bien peu le comprenaient ", in Georges Bouchard, art. cit., p. 41.

25. Archives Départementales de la Côte-d'Or, C 3413.

26. Gabriel DUMAY, « Une session des États généraux de Bourgogne à Autun en 1763 ", Mémoires de la société éduenne, VIII, 1879 ; François Dumont, Une session des États de Bourgogne. La tenue de 1718, Dijon, Bernigaud et Privat, 1935 ; Félix Bolley, «La session de 1787 des États de Bourgogne », Mémoires pour l'histoire du droit et des institutions des anciens pays bourguignons, comtois et romands, 1938, p. 200-2004; Daniel LIGou, " La dernière session des États de Bourgogne (1787): représentation et conflit d'ordres", Herrschaftsverträge, Wahlkapitulationen, Fundamentalgesetze, Göttingen, Vandenhoeck-Ruprecht, 1977, p. 121-130.

27. Christian DUGAS DE LA BOISSONNY, «Les voyages d'honneur des États de Bourgogne de 1682 à 1785 », Annales de l'Est, 1986, p. 251-308.

28. Christine LAMARRE, « Les présents en vins des villes et États de Bourgogne », Dix-huitième siècle, n²9, 1997, p. 125-136. 
29. Daniel LIGOU, «Le personnel des ponts et chaussées en Bourgogne au XVIII ${ }^{\mathrm{e}}$ siècle ", Actes du $96^{e}$ Congrès National des Sociétés Savantes, Toulouse, Paris, Bibliothèque nationale, 1978, p. 337-364.

30. Daniel LIGOU, «La province et son armature », in Histoire de la Bourgogne, dir. Jean Richard, Toulouse, Privat, 1978, p. 269-306.

31. Notamment celle d'Arthur KLEINCLAUSZ, Histoire de la Bourgogne, Paris, Hachette, 1909, 453 p.

32. James Russell MAJOR, Representative government in Early Modern France, New-Haven and London, Yale University Press, 1980, $731 \mathrm{p}$.

33. James Russell MAJOR, From Renaissance Monarchy to Absolute Monarchy. French kings, nobles and Estates, Baltimore and London, The John Hopkins University Press, 1994, 444 p.

34. Mark POTTER et Jean-Laurent ROSENTHAL, «The burgundian Estates' bond market : clienteles and intermediaries, 1660-1790 ", in Des personnes aux institutions : réseaux et cultures du crédit du $16^{e}$ au $20^{e}$ siècles en Europe, éd. Laurence Fontaine, Gilles Postel-Vinay, Jean-Laurent Rosenthal et Paul Servais, Louvain-la-Neuve, Academia Bruylant, 1997, p. 173-195 et « Politics and public finance in France : the Estates of Burgundy, 1660-1790 », The journal of interdisciplinary history, 1997, vol. 27, n ${ }^{\circ}$, p. p77-612.

35. Mark POTTER et Jean-Laurent ROSENTHAL, «The developpement of Intermediation French Credit Markets : Evidence from the Estates of Burgundy ", Journal of economic history, vol. $62, \mathrm{n}^{\circ} 4$, dec. 2002, p. 1030.

36. Mark POTTER, Corps and clienteles. Public finance and political change in France, 1688-1715, Alderhot, Ashgate, 2003, p. 26.

37. Sur cette question voir en particulier le chapitre III : "Estates and ruling coalitions in Burgundy ".

38. Ibid., p. 98.

39. Ibid., p. 192. Pour un résumé de la thèse voir : Mark POTTER, «Coalitions and Local Politics in seventeenth-century France », French Historical Studies, vol. 31, n²1, 2008, p. 29-49.

40. Alexis de TOCQUEVILLE, L'Ancien Régime et la Révolution, Paris, Lévy, 1856, chapitre XI, «De l'espèce de liberté qui se rencontrait sous l'Ancien Régime et de son influence sur la Révolution ».

41. Ernest LAVISSE, Louis XIV, Paris, Hachette, 1908 ; rééd. R. Laffont, 1989, p. 259.

42. Beth NACHISON, Provincial government in the Ancien Regime: The Princes of Condé in Burgundy, 1660-1730, Phd, The University of Iowa, 1992.

43. Beth NACHISON, "Absentee Government and Provincial Governors in Early Modern France : The Princes of Condé and Burgundy, 1660-1720", French Historical Studies, vol. 21, n², 1998, p. 265-297.

44. Julian SWANn, Provincial Power and Absolute Monarchy. The Estates Général of Burgundy, 1661-1790, Cambridge, C.U.P., 2003, 460 p.

45. William BEIK, Absolutisme and Society in Seventeenth-Century France. State power and Provincial Aristocracy in Languedoc, Cambridge, C.U.P., 1985, 375 p.

46. Julian SWANN, op.cit., p. 133.

47. Ibid., p. 151.

48. Ibid., chap. 11 : « An enlightened administration?».

49. Ibid., p. 300.

50. Ibid., p. 411.

51. Charles PAPON, Le système financier bourguignon dans la première moitié du XVIII ${ }^{e}$ siècle, ClermontFerrand, Fondation Varenne, 2007, $647 \mathrm{p}$.

52. Charles PAPON, Le système financier bourguignon dans la première moitié du XVIII siècle (1710-1752), thèse pour le doctorat en droit, sous la dir. d'Albert Rigaudière, Paris II, octobre 2005, p. 157.

53. Ibid., p. 233.

54. Ibid., p. 243.

55. Ibid., p. 270. 
56. Ibid., p. 274.

57. Ibid., p. 323.

58. Stéphane PANNEKOUCKE, Des princes en Bourgogne. Les Condé gouverneurs au XVIII ${ }^{e}$ siècle, Paris, éd du C.T.H.S., 2010, 338 p.

59. Ibid., chap. 5 : « Le gouverneur et la province, une médiation institutionnalisée ».

60. Ibid, p. 147-148.

\section{AUTEUR}

\section{JÉRÔME LOISEAU}

Maître de conférences en histoire moderne, Laboratoire des Sciences Historiques (LSH, EA 2273), Université de Franche-Comté, 30-32 rue Mégevand, 25030 Besançon Cedex 